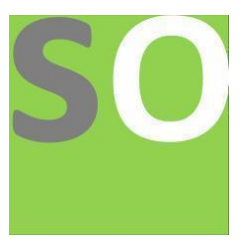

Article title: Solution of Lane Emden-Fowler Partial Differential Equations by Taylor

Strategy $\quad$ Series

Authors: tarig elzaki[1]

Affiliations: University of Jeddah[1]

Orcid ids: 0000-0002-6946-9267[1]

Contact e-mail: tarig.alzaki@gmail.com

License information: This work has been published open access under Creative Commons Attribution License $\mathrm{http}: / / c r e a t i v e c o m m o n s . o r g / l i c e n s e s / b y / 4.0 /$, which permits unrestricted use, distribution, and reproduction in any medium, provided the original work is properly cited. Conditions, terms of use and publishing policy can be found at https://www.scienceopen.com/.

Preprint statement: This article is a preprint and has not been peer-reviewed, under consideration and submitted to ScienceOpen Preprints for open peer review.

DOI: 10.14293/S2199-1006.1.SOR-.PPZP6RC.v1

Preprint first posted online: 07 January 2022

Keywords: Taylor series, , Lane Emden-Fowler PDEs, , Lane-Emden type equations. 


\title{
Solution of Lane Emden-Fowler Partial Differential Equations by Taylor Series Strategy
}

\author{
Tarig M. Elzakia, Mourad Chamekh ${ }^{\text {ab, }}$,Shams A. Ahmed ${ }^{\mathrm{c}}$, \\ ${ }^{a}$ Mathematics Department, College of Sciences and Arts, Alkamel, University of Jeddah, Saudi Arabia \\ ${ }^{a b}$ University of Tunis El Manar, National Engineering School at Tunis, LAMSIN, 1002, Tunis, Tunisia \\ ${ }^{c}$ Department of Mathematic, Faculty of Sciences and Arts, Jouf University, Tubarjal, Saudi Arabia \\ ${ }^{c}$ Department of Mathematic, University of Gezira, Sudan \\ atarig.alzaki@gmail.com, $, \underline{\text { tffarah@uj.edu.sa }},{ }^{\text {ab }} \underline{\text { chamekhmourad1@gmail.com }} \&^{\mathrm{c}} \underline{\text { shamsaldeenahmed@ gmail.com }}$
}

\begin{abstract}
The Taylor series scheme is one of the most primitive analytic-numeric algorithms for exact solution of initial value problems for deferential equations. Taylor's series technique is a simple and effective tool for dealing with some PDEs. The main idea of the treatment of these algorithms is based on the calculation of higher derivatives using well-known technique for the Lane Emden-Fowler PDEs. Therefore, this paper is directed to show this method of solving Lane Emden-Fowler PDEs with singular boundary value problems, we found that this strategy is rarely used. The numerical results obtained demonstrate that: this technique is exceptionally simple and valuable compared to other strategies.
\end{abstract}

\section{Keywords}

Taylor series, Lane Emden-Fowler PDEs, Lane-Emden type equations.

\section{Introduction}

The Taylor series algorithm is one of the most primitive algorithms for the approximate or exact solution for initial value problems for differential equations, Lane-Emden-Fowler equations (LEFE) are one of the foremost spurring and most fundamental equations because it portrays an assortment of problems in dynamics and physics; also LEFE (Atta Ullah et al., 2018), has many applications in astrophysics and engineering sciences. Lane-Emden's equation has essential value in the modern analysis of several harms in astrophysics and relativity together with a number of models of density profiles for dark matter halos.

Solutions to these kinds of problems provide a few uncommon solutions that are closely related to LEFE. This type of problem has been utilized in different areas like engineering, physics, and dynamics (Chowdhury et al., 2007). Many researchers have utilized distinct techniques such as the He's method (J.-H. He, 1999, 2000, 2003, 2005), the semi-inverse method (Heydari M.et al., 2011) and the homotopy perturbation method (Othman et al., 2010) and (Babolian E. et al., 2009) to find the solutions of the LEFE.

Present numerical algorithms for the solution of differential equations are also based on the technique of the Taylor series. Each algorithm, such as the Runge-Kuttaor the multistep methods are construct so that they give a phrase depending on a parameter (h) called step size as an 
approximate solution and the first terms of the Taylor series of this phrase must be matching with the terms of the Taylor series of the exact solution.

The Taylor series is valuable ways of writing functions in the form of an infinite sum of terms that are calculate from the values of derivatives of a function at one point.

\section{Definition 1.1}

If $\Phi(x, t)$ is a function of two variables have a second partial derivatives at the point $(\alpha, \beta)$, then Taylor series is,

$$
\begin{aligned}
& \Phi_{2}(x, t)=\Phi(\alpha, \beta)+\Phi_{x}(\alpha, \beta)(x-\alpha)+\Phi_{t}(\alpha, \beta)(t-\beta)+\frac{\Phi_{x x}(\alpha, \beta)}{2}(x-\alpha)^{2} \\
& +\Phi_{x t}(\alpha, \beta)(x-\alpha)(t-\beta)+\frac{\Phi_{t t}(\alpha, \beta)}{2}(t-\beta)^{2}
\end{aligned}
$$

\section{Definition 1.2}

If $\Phi(x, t)$ is a function of two variables have a second partial derivatives at the point $(\alpha, \beta)$, then $n^{\text {th }}$ degree Taylor series is,

$\Phi_{n}(x, t)=\sum_{i=0}^{n} \sum_{j=0}^{n-i} \frac{\partial^{(i+j)} \Phi(\alpha, \beta)}{\partial x^{i} \partial t^{j}} \frac{(x-\alpha)^{i}(t-\beta)^{j}}{i ! j !}$.

It is easy to find $\Phi_{2}(x, t), \Phi_{3}(x, t), \Phi_{4}(x, t), \ldots, \Phi_{n}(x, t)$, from Eq. (2) if we need, for example,

$$
\begin{gathered}
\Phi_{3}(x, t)=\Phi_{2}(x, t)+\frac{\Phi_{x x x}(\alpha, \beta)}{6}(x-\alpha)^{3}+\frac{\Phi_{t x x}(\alpha, \beta)}{2}(x-\alpha)^{2}(t-\beta) \\
+\frac{\Phi_{t t x}(\alpha, \beta)}{2}(x-\alpha)(t-\beta)^{2}+\frac{\Phi_{t t t}(\alpha, \beta)}{6}(t-\beta)^{3} .
\end{gathered}
$$

In this paper, we utilize the Taylor series strategy in two variables for the exact solutions form of the LEFEis as follow,

$$
\Phi_{x x}(x, t)+\frac{k}{x} \Phi_{x}(x, t)+f(x, t) \Phi(x, t)=\Phi_{t t}(x, t)+h(t) g(x) \text {. }
$$

With the boundary conditions,

$\Phi(0, t)=\delta_{1}(t), \Phi_{x}(0, t)=\delta_{2}(t)$,

\section{Description of Strategy}

This strategy is based on the initial or boundary conditions of equations (4) and (5) and the Taylor series given by equation (2), this is often done by finding this series at the initial conditions and after that finding the exact solution of equations(4), (5) which may be convergent or non-convergent.

In the order to give the Taylor series, we need to find $\Phi_{x x}(0, t), \Phi_{x x x}(0, t), \cdots$. and then substitute them in Eq. (2). For this, considering the equation (4), can be written as:

$$
x \Phi_{x x}+k \Phi_{x}+x f \Phi=x \Phi_{t t}+x h(t) g(x)
$$


With: $f=f(x, t), \Phi=\Phi(x, t)$ and the real $k \neq-1$.

By differentiating with respect $\mathrm{x}$ of the equation (6), we obtain:

$$
\Phi_{x x}+x \Phi_{x x x}+k \Phi_{x x}+f \Phi+x f_{x} \Phi+x f \Phi_{x}=\Phi_{t t}+x \Phi_{x t t}+h(t) g(x)+x h(t) g^{\prime}(x) \text {. }
$$

Put $x=0$, we obtain:

$$
\Phi_{x x}(0, t)+k \Phi_{x x}(0, t)+f(0, t) \Phi(0, t)=\Phi_{t t}(0, t)+h(t) g(0) .
$$

Thanks to the boundary conditions, we can write,

$$
\left.\Phi_{x x}(0, t)=\frac{1}{1+k}\left[\delta_{1}^{\prime \prime}(t)+h(t) g(0)-f(0, t) \delta_{1}(t)\right)\right]
$$

We repeat again the differentiating with respect to $\mathrm{x}$ of the equation (7), we obtain:

$\Phi_{x x x}+\Phi_{x x x}+x \Phi_{x x x x}+k \Phi_{x x x}+f_{x} \Phi+f \Phi_{x}+f_{x} \Phi+x f_{x x} \Phi+x f_{x} \Phi_{x}+f \Phi_{x}+x f_{x} \Phi_{x}+$ $x f \Phi_{x x}=\Phi_{x t t}+x \Phi_{x x t t}+\Phi_{x t t}+2 h(t) g^{\prime}(x)+x h(t) g^{\prime \prime}(x)$.

For, $x=0$, we find that:

$$
\Phi_{x x x}(0, t)=\frac{2}{2+k}\left[\delta_{2}^{\prime \prime}(t)+h(t) g^{\prime}(0)-f_{x}(0, t) \delta_{1}(t)-f(0, t) \delta_{2}(t)\right] .
$$

Then, we obtain an expression, when all the variables are well known.

In the same way we obtain the other partial derivatives.

\section{Applications}

To demonstrate this strategy, we'll present some different examples of some Lane Emden-Fowler PDEs.

\section{Example 1}

Consider the following LEFE,

$$
\Phi_{x x}+\frac{2}{x} \Phi_{x}-\left(5+4 x^{2}\right) \Phi=\Phi_{t}-4 x^{4}-5 x^{2}+6
$$

With the boundary conditions,

$\Phi(0, t)=e^{t}, \Phi_{x}(0, t)=0$,

To find $\Phi_{x x}(0, t), \Phi_{x x x}(0, t), \ldots$ and then substitute them in Eq. (2),

We can write Eq. (8) in the form:

$$
x \Phi_{x x}+2 \Phi_{x}-\left(5 x+4 x^{3}\right) \Phi=x \Phi_{t}-4 x^{5}-5 x^{3}+6 x
$$

Differentiate this equation with respects to $x$,we obtain, $x \Phi_{x x x}+3 \Phi_{x x}-\left[\left(5 x+4 x^{3}\right) \Phi_{x}+\left(5+12 x^{2}\right) \Phi\right]=x \Phi_{t x}+\Phi_{t}-20 x^{4}-15 x^{2}+6$ 
Put $x=0$, to obtain:

$3 \Phi_{x x}(0, t)-5 \Phi(0, t)=\Phi_{t}(0, t), \Rightarrow 3 \Phi_{x x}(0, t)=e^{t}+6+5 e^{t}, \Rightarrow \Phi_{x x}(0, t)=2 e^{t}+2$,

We repeat this again and put $x=0$, to obtain:

$$
\Phi_{x x x}(0, t)=0, \Phi_{x x x x}(0, t)=12 e^{t}, \Phi_{x x x x x}(0, t)=0, \ldots
$$

Lastly substitute in Eq. (2), to find:

$\Phi(x, t)=e^{t}+\frac{x^{2}}{2 !}\left(2 e^{t}+2\right)+12 e^{t} \frac{x^{4}}{4 !}+\ldots .=x^{2}+e^{t}\left(1+x^{2}+\frac{x^{4}}{2 !}+\ldots.\right)$,

Whichis converges to the exact solution:

$$
\Phi(x, t)=x^{2}+e^{t+x^{2}}
$$

Note that: we can solve this problem by using the initial condition using the same method.

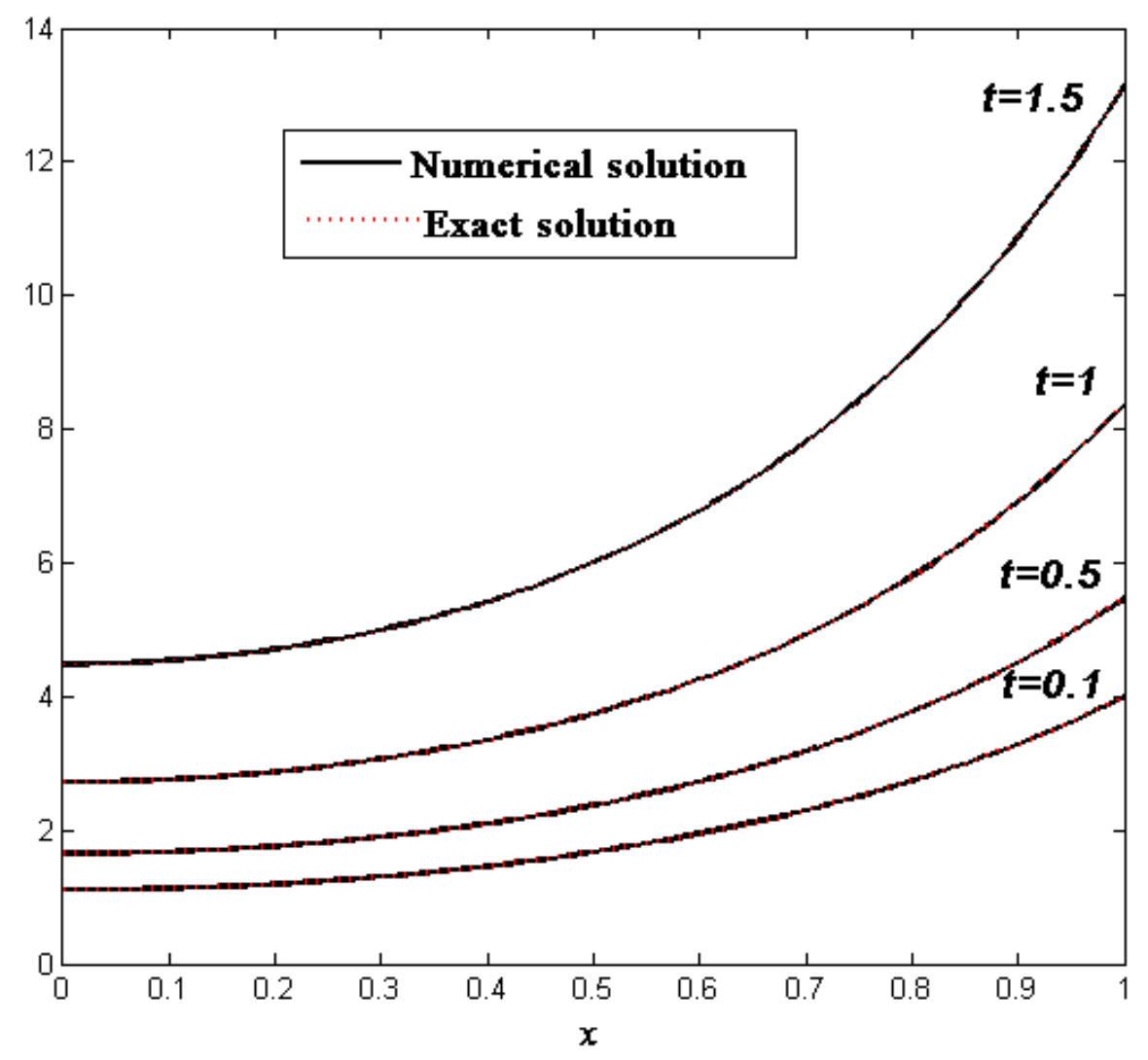

Figure 1 Solution of example 1 


\section{Example 2}

Consider the linear LEFE,

$$
\begin{aligned}
& \Phi_{x x}+\frac{2}{x} \Phi_{x}-\left(5+4 x^{2}\right) \Phi=\Phi_{t t}-4 x^{5}-5 x^{3}+12 x, o r \\
& x \Phi_{x x}+2 \Phi_{x}-\left(5 x+4 x^{3}\right) \Phi=x \Phi_{t t}-4 x^{6}-5 x^{4}+12 x^{2}
\end{aligned}
$$

With the boundary conditions,

$$
\Phi(0, t)=\cosh t, \Phi_{x}(0, t)=0,
$$

Using the same steps that we used in example 1,

$$
\begin{aligned}
& x \Phi_{x x x}+3 \Phi_{x x}-\left[\left(5 x+4 x^{3}\right) \Phi_{x}+\left(5+12 x^{2}\right) \Phi\right]=x \Phi_{t t x}+\Phi_{t t}+24 x-20 x^{3}-24 x^{5}, \\
& 3 \Phi_{x x}(0, t)-5 \cosh t=\cosh t, \Rightarrow \Phi_{x x}(0, t)=2 \cosh t \\
& x \Phi_{x x x x}+4 \Phi_{x x x}-\left[\left(5 x+4 x^{3}\right) \Phi_{x x}+\left(5+12 x^{2}\right) \Phi_{x}+\left(5+12 x^{2}\right) \Phi_{x}+24 x \Phi\right] \\
& =x \Phi_{t t x x}+\Phi_{t t x}+24-60 x^{2}-120 x^{4}, \\
& 4 \Phi_{x x x}(0, t)=24 \Rightarrow \Phi_{x x x}(0, t)=6, \Phi_{x x x x}(0, t)=12 \cosh t,
\end{aligned}
$$

Then the solution step-by-step converges to the closed form of the solution of problem(10-11), and we obtain,

$$
\Phi(x, t)=\cosh t+x^{2} \cosh t+x^{3}+\frac{x^{4}}{4 !} 12 \cosh t+\ldots ., \Rightarrow \Phi(x, t)=x^{3}+e^{x^{2}} \cosh t
$$

Also, we can solve this problem by using the initial condition using the same method. 


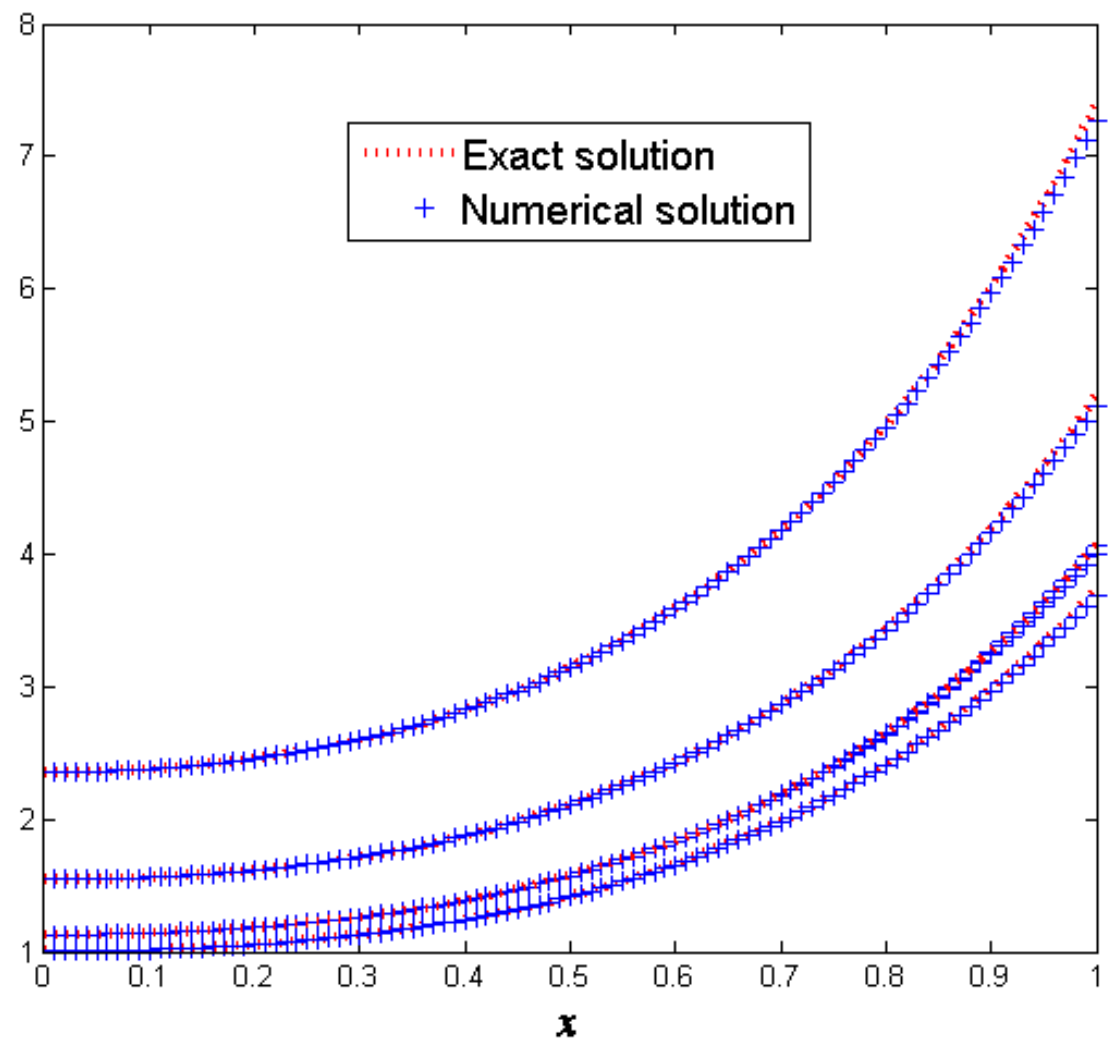

Figure 2 solution of example 2

\section{Discussion}

In the fig. 1 and fig. 2, we present the numerical solutions obtained after 3 steps of the Taylor procedure using different values of $t=0.1 ; 0.5 ; 1$ and 1.5 . We notice that the convergence towards a precise solution is fast and is done in a few steps.

\section{Conclusion}

The Taylor series method with numerical derivatives proposed in this article is such algorithm which can be spirited with the classical algorithm for exact solution of LEFE with singular boundary value problems, from the prepared illustrations; we watched that the solutions gotten by the Taylor series strategy are precise and quick.

Also, this procedure is valuable and straightforward and does not require a complex calculation compared to other strategies. In the forthcoming studies, we are going attempt to expand the utilized of the Taylor series method to the integro-differential equations.

\section{Acknowledgment}

This work was funded by the University of Jeddah, Jeddah, Saudi Arabia, under grant No. (UJ21-DR-144). The authors, therefore, acknowledge with thanks the University of Jeddah technical and financial support. 


\section{References}

Atta Ullah, Kamal Shah, Numerical analysis of Lane Emden - Fowler equations. Journal of Taibah University for Science. 2018; 12 (2): 180 - 185.

Babolian E, Azizi A, Saeidian J. Some notes on using the homotopy perturbation method for solving time dependent differential equations. Math Comput Model. 2009, 50:213-224.

Chowdhury MSH, Hashim I. Solutions of a class of singular second-order IVPs by homotopyperturbation method. Phys Lett. A. 2007; 365(5):439-447.

Heydari M, Hosseini SM, Loghmani GB. Numerical solution of singular IVPs of Lane-Emden type using integral operator and radial basis functions. Int J Indust Math.2011; 4(2):135-146.

J.-H. He, Homotopy perturbation method: a new nonlinear analytical technique, Appl. Math. Comput. 135 (2003), 73-79.1, 4.

J.-H. He, Homotopy perturbation technique, Comput. Methods Appl. Mech. Engrg., 178 (1999), 257-262. 1, 4.

J.-H. He, Variational iteration method-a kind of non-linear analytical technique: some examples, Internat. J. Non-Linear Mech., 34 (1999), 699-708. 1.

J.-H. He, Variational iteration method for autonomous ordinary differential systems, Appl. Math. Comput.114 (2000), 115-123. 1.

J.-H. He, Application of homotopy perturbation method to nonlinear wave equations, Chaos Solitons Fractals, 26 (2005),695-700. 1, 4.

Othman MIA, Mahdy AMS, Farouk RM. Numerical solution of 12th order boundary value problems by using homotopy perturbation method. J Math Comput Sci. 2010;1(1):14-27.

Richardson OU. The emission of electricity from hot bodies. London: Zongmans Green and Company; 1921. 